ASYMPTOTIC BEHAVIOR OF SOLUTIONS OF COAGULATION-FRAGMENTATION MODELS

By
Avner Friedman
and

Fernando Reitich

IMA Preprint Series \# 1479

June 1997

INSTITUTE FOR MATHEMATICS AND ITS APPLICATIONS

UNIVERSITY OF MINNESOTA

514 Vincent Hall

206 Church Street S.E.

Minneapolis, Minnesota 55455 


\title{
ASYMPTOTIC BEHAVIOR OF SOLUTIONS OF COAGULATION-FRAGMENTATION MODELS
}

\author{
AVNER FRIEDMAN* AND FERNANDO REITICH ${ }^{\dagger}$
}

\begin{abstract}
In this paper we study the evolution of the number density $n(x, c, t)$ of droplets of volume $x$ with chemical concentration $c$ subject to both coalescence (coagulation) and rupture (fragmentation, breakage). It is proved that as $t \rightarrow \infty$ the concentration tends to a limit value $c_{\infty}$ and the measure $x n(x, c, t) d x d c$ converges to $\delta\left(c-c_{\infty}\right) d L(x)$ where $\delta(y)$ is the Dirac measure and $d L$ is a measure satisfying the appropriate equilibrium equation.
\end{abstract}

1. The model. Consider a mixture of a large number of incompressible spherical droplets of varying volume size $x$ in a solution (e.g., water or air). When the mixture is well stirred the distribution of droplet size can be taken to be homogeneous in space. We may then introduce the notion of density number $n(x)$ :

$$
n(x) d x=\text { number of droplets in unit volume }
$$

whose volume size lies between $x$ and $x+d x$;

$n(x)$ is independent of the location of the volume element.

Since the droplets are in continuous motion, they may collide and, as a result, coalesce. The motion may also cause rupture. We assume that if two droplets of volumes $x$ and $\xi$ coalesce, they will form a droplet of volume $x+\xi$. The rate of coalescence is given by a function $K(x, \xi)$, which is called the coalescence or coagulation kernel.

We also introduce the fragmentation or breakage kernel $B(x, \xi)$ which is the rate that a droplet of volume $x$ breaks into two droplets of volumes $\xi$ and $x-\xi$.

Throughout this paper we assume that

$$
\begin{aligned}
& K(x, \xi) \text { is continuous and } \geq 0 \text { for } x, \xi \geq 0, \\
& K(x, \xi)=K(\xi, x), \\
& K(x, \xi) \leq C_{0}, \\
& B(x, \xi) \text { is continuous and } \geq 0 \text { for } x \geq \xi \geq 0, \\
& B(x, \xi)=B(x, x-\xi), \\
& \int_{0}^{x} B(x, \xi) d \xi \leq C_{1}, \\
& \int_{0}^{x} \xi B(x, \xi) d \xi \leq C_{2} x .
\end{aligned}
$$

Remark 1.1. Note that if volume is preserved under rupture, then

$$
\int_{0}^{x} B(x, \xi) \xi d \xi=x
$$

\footnotetext{
* University of Minnesota, Institute for Mathematics and its Applications, Minneapolis, Minnesota 55455.

${ }^{\dagger}$ Department of Mathematics and Center for Research in Scientific Computation, North Carolina State University, Box 8205, Raleigh, NC 27695-8205.
} 
so that the last condition in (1.1) is very natural.

Due to coagulation and fragmentation the number density will actually vary with time, and we introduce

$$
n(x, t)=\text { number density at time } t \text {. }
$$

The following conservation law holds:

$$
\begin{aligned}
& \frac{d}{d t} n(x, t) d x=\text { number gained through coalescence } \\
& \quad+\text { number gained through rupture } \\
& \quad-\text { number lost through coalescence } \\
& \quad-\text { number lost through rupture. }
\end{aligned}
$$

This can be expressed by the evolution equation:

$$
\begin{aligned}
\frac{d}{d t} n(x, t) & =\frac{1}{2} \int_{0}^{x} K(x-\xi, \xi) n(x-\xi, t) n(\xi, t) d \xi+\int_{x}^{\infty} B(\xi, x) n(\xi, t) d \xi \\
& -n(x, t) \int_{0}^{\infty} K(x, \xi) n(\xi, t) d \xi-n(x, t) \int_{0}^{x} \frac{\xi}{x} B(x, \xi) d \xi
\end{aligned}
$$

Here the underlying assumption is that, although $n(x, t)$ varies with $t$, the mixture is maintained homogeneous in space at any moment (due to continuous fast stirring).

Together with (1.2) we prescribe an initial condition

$$
n(x, 0)=n_{0}(x), \quad n_{0}(x) \geq 0 .
$$

There is substantial literature on coagulation and coagulation-fragmentation models. Melzak [10] proved a general existence, uniqueness and positivity of a solution for all $t>0$. For other results we refer to [2], [4], [9] and the references therein.

We shall actually be interested in a situation where each droplet carries a chemical species which is uniformly distributed within it; the concentration $c$ of the chemical species is a variable quantity. We then introduce

$$
\begin{aligned}
& n(x, c, t) d x d c=\text { number of droplets at time } t \\
& \text { with volume size in the interval }(x, x+d x) \\
& \text { and concentration (of the chemical } \\
& \text { species) in the interval }(c, c+d c) .
\end{aligned}
$$


Analogously to (1.2), the number density $n(x, c, t)$ evolves as follows:

$$
\begin{aligned}
\frac{d}{d t} n(x, c, t) & =\frac{1}{2} \int_{0}^{x} \int_{0}^{\infty} K(x-\xi, \xi) n\left(x-\xi, \frac{x c-\xi \gamma}{x-\xi}, t\right) n(\xi, \gamma, t) \frac{x}{x-\xi} d \gamma d \xi \\
& +\int_{x}^{\infty} B(\xi, x) n(\xi, c, t) d \xi \\
& -n(x, c, t) \int_{0}^{\infty} \int_{0}^{\infty} K(x, \xi) n(\xi, \gamma, t) d \gamma d \xi \\
& -n(x, c, t) \int_{0}^{x} \frac{\xi}{x} B(x, \xi) d \xi
\end{aligned}
$$

where we define

$$
n\left(x, c^{\prime}, t\right)=0 \quad \text { if } c^{\prime}<0 .
$$

Here we used the fact that rupture does not change the concentration. On the other hand, if particles $(\xi, \gamma)$ and $(\eta, \beta)$ coalesce to produce $(x, c)$, then

$$
x=\xi+\eta, \quad x c=\xi \gamma+\eta \beta
$$

so that

$$
\eta=x-\xi, \quad \beta=\frac{x c-\xi \gamma}{x-\xi}
$$

and $d \gamma d \xi d \eta d \beta=d \gamma d \xi \frac{x}{x-\xi} d x d c$, since the Jacobian determinant

$$
\left|\begin{array}{ll}
\frac{\partial \eta}{\partial x} & \frac{\partial \eta}{\partial c} \\
\frac{\partial \beta}{\partial x} & \frac{\partial \beta}{\partial c}
\end{array}\right| \text { is equal to } \frac{x}{x-\xi}
$$

this explains the appearance of $x /(x-\xi)$ in the first integral on the right hand side of (1.4).

Remark 1.2. The last integral on the right-hand side of (1.2), or (1.4), can also be written in the form

$$
\frac{1}{2} \int_{0}^{x} B(x, \xi) d \xi
$$

since

$$
\begin{aligned}
\frac{1}{2} \int_{0}^{x} B(x, \xi) d \xi & =\frac{1}{2} \frac{1}{x} \int_{0}^{x}[\xi B(x, \xi)+(x-\xi) B(x, \xi)] d \xi \\
& =\frac{1}{2} \frac{1}{x}\left[\int_{0}^{x} \xi B(x, \xi) d \xi+\int_{0}^{x}(x-\xi) B(x, x-\xi) d \xi\right] \\
& =\frac{1}{x} \int_{0}^{x} \xi B(x, \xi) d \xi .
\end{aligned}
$$


Together with (1.4) we prescribe an initial condition

$$
n(x, c, 0)=n_{0}(x, c), \quad n_{0}(x, c) \geq 0 .
$$

The model (1.4) arises in the process of making a photographic film. The film is made of several emersion layers. One of them consists of oil droplets in aqueous solution. The mixture, prior to being coated on the film substrate, is subjected to coalescence and rupture by means of stirring. A key problem is to determine the rate of coalescence (which depends on parameters, such as the amount of surfactant added at the initial time). One of the methods to determine coalescence is based on adding chemilumenescent species to the mixture:

The optical signal, which is the number of photons emitted per unit time from a droplet, is a function, $f(x, c)$, of the species concentration $c$ in the droplet and the droplet volume $x$. One can measure the total signal

$$
S(t)=\int_{0}^{\infty} \int_{0}^{\infty} f(x, c) n(x, c) d x d c
$$

and this is used to to evaluate the rate of coalescence [8, Chap. 6].

Note that if two drops with parameters $\left(x_{1}, c_{1}\right)$ and $\left(x_{2}, c_{2}\right)$ coalesce to form a drop with parameters $(\tilde{x}, \tilde{c})$ then

$$
\tilde{x}=x_{1}+x_{2}, \quad \tilde{c}=\frac{x_{1} c_{1}+x_{2} c_{2}}{x_{1}+x_{2}} .
$$

Typically $f(x, c)=x c^{2}$, and then

$$
f(\tilde{x}, \tilde{c})<f\left(x_{1}, c_{1}\right)+f\left(x_{2}, c_{2}\right)
$$

so that the total signal should decrease.

The model (1.4) was developed by David Ross; see [8, Chap. 6]. He conjectured that as $t \rightarrow \infty$ the concentrations $c$ will all tend to a constant, say $c_{\infty}$. With this in mind, one defines the degree of coalescence

$$
D(t)=\int_{0}^{\infty} \int_{0}^{\infty}\left(c-c_{\infty}\right)^{2} x n(x, c, t) d x d c
$$

and one would like to prove rigorously that

$$
D(t) \rightarrow 0 \text { if } t \rightarrow \infty
$$

Consider next the measures $d \mu_{t}$ defined by

$$
d \mu_{t}(x, c)=x n(x, c, t) d x d c
$$

If (1.8) is true then we may go one step further and try to prove that as $t \rightarrow \infty$

$$
d \mu_{t}(x, c) \rightarrow \delta\left(c-c_{\infty}\right) d L(x)
$$

in the sense of weak convergence of measures, where $\delta(y)$ is the Dirac measure and

$d L$ is a solution of the equilibrium equation (1.2). 
In this paper we shall prove, under mild additional conditions on $K$ and $B$ that (1.8) holds. We shall also establish $(1.10),(1.11)$ under the additional condition that

$$
\begin{aligned}
& K(x, \xi) \leq \eta(x+\xi), \quad \gamma_{0} \equiv \inf _{x>0} \frac{1}{x} \int_{0}^{x} \xi B(x, \xi) d \xi>0, \text { where } \\
& 2 \eta \int_{0}^{\infty} \int_{0}^{\infty} x n_{0}(x, c) d x d c<\gamma_{0} .
\end{aligned}
$$

The structure of the paper is as follows:

In $\S 2$ we establish existence, uniqueness and positivity of the solution of (1.4), (1.5) for all $t>0$. Assuming that

$$
n_{0}(x)=\int_{0}^{\infty} n_{0}(x, c) d c
$$

we also prove that the function

$$
n(x, t)=\int_{0}^{\infty} n(x, c, t) d c
$$

is the unique solution of $(1.2),(1.3)$. In $\S 3$ we prove conservation of mass

$$
\int_{0}^{\infty} x n(x, t) d x=\text { const. }
$$

We also show that if $n_{0}(x, c)=0$ whenever $c>c_{*}\left(c_{*}\right.$ constant) then $n(x, c, t)=0$ for $c>c_{*}$. Using these results we derive in $\S 4$ a formula for $d S / d t$ (see (4.2)) when $S$ is defined in (1.6) and $f(x, c)$ is any function satisfying $|f(x, c)| \leq C(1+x)$. This formula is the key to the proofs of both (1.8) (in $\S 5)$ and (1.10), (1.11) (in $\S 6$ ).

We finally remark that a coalescence problem for $n(x, c, t)$ was studied in [1], with $c$ being the concentration of surfactant which inhibits coalescence. The limit behavior of the solution and the mathematical method for deriving it are entirely different from the limit behavior and the methods of the present paper.

2. Existence and uniqueness. We shall need the following assumptions:

$$
\begin{aligned}
& n_{0}(x) \text { is continuous for } x \geq 0, \\
& 0 \leq n_{0}(x) \leq A_{0}, \quad \int_{0}^{\infty} n_{0}(x) d x \leq A_{0}, \\
& n_{0}(x, c) \text { is continuous for } x \geq 0, c \geq 0, \\
& 0 \leq n_{0}(x, c) \leq A_{1}, \quad \int_{0}^{\infty} \int_{0}^{\infty} n_{0}(x, c) d x d c \leq A_{1}
\end{aligned}
$$

where $A_{0}, A_{1}$ are positive constants. 
THEOREM 2.1. If (1.1), (2.1) hold then there exists a unique solution of (1.2), (1.3) with $n(x, t), \partial n(x, t) / \partial t$ continuous for $x \geq 0, t \geq 0$, such that, for each $T>0$,

$$
\sup _{x \geq 0}|n(x, t)|+\int_{0}^{\infty}|n(x, t)| d x \leq C(T)<\infty \text { if } 0 \leq t \leq T .
$$

The solution has the following additional properties:

$$
\begin{aligned}
& n(x, t) \text { is analytic in } t, t \geq 0, \\
& n(x, t) \geq 0 .
\end{aligned}
$$

This theorem is due to Melzak [10]. We note that if $K$ and $B$ have compact support and $n_{0}(x)>0$ for all $x>0$, then the proof of (2.5) is much simpler than the proof given in [10] (or [3]). Indeed, if $x$ is large then from (1.2) we see that $d n(x, t) / d t=0$ so that $n(x, t)=n_{0}(x)>0$. Hence if (2.5) is not true, with strict inequality, then there is a smallest $t=t_{0}>0$ such that $n(x, t)>0$ if $t<t_{0}$ for all $x \geq 0$ and $n\left(x_{0}, t_{0}\right)=0$ for a finite point $x_{0}$. From (1.2) we then deduce that

$$
\frac{d n\left(x_{0}, t_{0}\right)}{d t}>0
$$

which is a contradiction. Finally, the solution $n(x, t)$ for general $K, B, n_{0}$, can be obtained as a pointwise limit of solutions with compact $K, B$ and strictly positive $n_{0}(x)$ (cf. [3] or [4]), so that $(2.5)$ is true in general.

THEOREM 2.2. If (1.1), (2.2) hold then there exists a unique solution of (1.4), (1.5) with $n(x, c, t), \partial n(x, c, t) / \partial t$ continuous for $x \geq 0, c \geq 0, t \geq 0$, such that, for each $T>0$,

$$
\sup _{x \geq 0, c \geq 0}|n(x, c, t)|+\int_{0}^{\infty} \int_{0}^{\infty}|n(x, c, t)| d x d c \leq C(T)<\infty \text { if } 0 \leq t \leq T .
$$

The solution has the following additional properties:

$$
\begin{aligned}
& n(x, c, t) \text { is analytic in } t, t \geq 0, \\
& n(x, c, t) \geq 0 .
\end{aligned}
$$

Proof. The proof is similar to the proof of Theorem 2.1 provided we can treat the first term on the right-hand side of (1.4) analogously to the first term on the right-hand side of (1.2); this means that we have to control the factor $x /(x-\xi)$ in the integrand.

Introduce the operator

$$
J(m, n)=\frac{1}{2} \int_{0}^{x} \int_{0}^{\infty} K(x-\xi, x) m\left(x-\xi, \frac{x c-\xi \gamma}{x-\xi}\right) \frac{x}{x-\xi} n(\xi, \gamma) d \gamma d \xi
$$

and break up the integral into

$$
\int_{0}^{x / 2} \int_{0}^{\infty}+\int_{x / 2}^{x} \int_{0}^{\infty}
$$


In the second integral substitute

$$
\gamma \rightarrow \gamma^{\prime}=\frac{x c-\xi \gamma}{x-\xi}, \quad \text { or } \gamma=\frac{x c-(x-\xi) \gamma^{\prime}}{\xi}, d \gamma=-\frac{x-\xi}{\xi} d \gamma^{\prime}
$$

We then obtain

$$
\begin{aligned}
J(m, n) & =\frac{1}{2} \int_{0}^{x / 2} \int_{0}^{\infty} K(x-\xi, \xi) m\left(x-\xi, \frac{x c-\xi \gamma}{x-\xi}, t\right) n(\xi, \gamma, t) \frac{x}{x-\xi} d \gamma d \xi \\
& -\frac{1}{2} \int_{x / 2}^{x} \int_{0}^{\infty} K(x-\xi, \xi) n\left(\xi, \frac{x c-(x-\xi) \gamma^{\prime}}{\xi}, t\right) m\left(x-\xi, \gamma^{\prime}, t\right) \frac{x}{\xi} d \gamma^{\prime} d \xi \\
& =J_{1}-J_{2} .
\end{aligned}
$$

Since the factors $\frac{x}{x-\xi}$ and $\frac{x}{\xi}$ in $J_{1}$ and $J_{2}$ respectively are uniformly bounded, we can easily establish, as in [10], the bound

$$
|J(m, n)|_{L^{\infty}} \leq C|n|_{L^{\infty}} \iint|m|+C|m|_{L^{\infty}} \iint|n|
$$

where

$$
\begin{aligned}
|f|_{L^{\infty}} & =\sup _{x \geq 0, c \geq 0}|f(x, c, t)| \\
\iint|f| & =\int_{0}^{\infty} \int_{0}^{\infty}|f(x, c, t)| d c d x .
\end{aligned}
$$

Next we estimate

$$
\iint|J(m, n)| d c d x
$$

by substituting

$$
c \rightarrow c^{\prime}=\frac{x c-\xi \gamma}{x-\xi}, \quad d c^{\prime}=\frac{x}{x-\xi} d c
$$

we obtain

$$
\iint|J(m, n)| \leq C \iint|m| \cdot \iint|n|
$$

With (2.9), (2.10) at hand, we can now proceed as in [10], with just minor changes, to establish Theorem 2.2. The positivity of $n(x, c, t)$ can be proved, more simply, by the argument following Theorem 2.1.

THEOREM 2.3. If (1.1), (2.1), (2.2) hold and, in addition,

$$
n_{0}(x)=\int_{0}^{\infty} n_{0}(x, c) d c
$$


then

$$
\int_{0}^{\infty} n(x, c, t) d c=n(x, t)
$$

where $n(x, c, t)$ and $n(x, t)$ are the solutions of (1.4), (1.5) and (1.2), (1.3), respectively.

Proof. We first proceed formally to integrate (1.4) with respect to $c$. In the first integral on the right-hand side we substitute

$$
c \rightarrow c^{\prime}=\frac{x c-\xi \gamma}{x-\xi}, d c^{\prime}=\frac{x}{x-\xi} d c
$$

to get

$$
\frac{1}{2} \int_{0}^{\infty} K(x-\xi, \xi) n(x-\xi, t) n(\xi, t) d \xi .
$$

where $n(x, t)$ is the function defined by the left-hand side of (2.12). All the other integrals yield immediately the corresponding integrals of (1.2). Hence, by uniqueness, the left-hand side of (2.12) is the solution of $(1.2),(1.3)$.

To prove the theorem rigorously we rewrite (1.4) in integrated form:

$$
n(x, c, t)-n_{0}(x, c)=\int_{0}^{t} d t\left\{\frac{1}{2} \int_{0}^{x} \int_{0}^{\infty} K \cdots+\cdots\right\} .
$$

Since

$$
\int_{0}^{\infty} \int_{0}^{\infty} n(x, c, t) d c d x \leq C(T)<\infty \text { if } 0 \leq t \leq T
$$

the integral

$$
\int_{0}^{\infty} n(x, c, t) d c
$$

is finite a.e. in $x$, for each $t$, and we may integrate (2.15) with respect to $c, 0 \leq c<\infty$. Each of the four multiple integrals thus obtained is well defined and is finite. We now proceed with the substitution (2.13) to obtain the term (2.14, and the proof of the theorem then follows as before.

\section{Conservation and invariant laws.}

Theorem 3.1. (Conservation law). If (1.1), (2.1) hold and

$$
\int_{0}^{\infty} x n_{0}(x) d x<\infty
$$


then

$$
\int_{0}^{\infty} x n(x, t) d x=\int_{0}^{\infty} x n_{0}(x) d x
$$

for all $t>0$.

Proof. The assertion (3.2) means that

$$
\frac{d}{d t} \int_{0}^{\infty} x n(x, t) d x=0
$$

This relation follows formally as a special case of Theorem 4.1 with $f(x, c)=x$. However, in order to apply the proof rigorously we need to know a priori that, for any $T>0$,

$$
\int_{0}^{\infty} x n(x, t) d x \leq C(T)<\infty \quad \text { if } \quad 0 \leq t \leq T .
$$

If $K, B$ and $n_{0}$ have compact support then (3.4) is true and then so is (3.2). We now approximate general $K, B, n_{0}$ by $K_{j}, B_{j}, n_{0, j}$ with compact support and denote the corresponding solutions by $n_{j}(x, t)$. Then, on one hand we have

$$
\int_{0}^{\infty} x n_{j}(x, t) d x=\int_{0}^{\infty} x n_{0, j}(x) d x \leq C
$$

for all $j$, and on the other hand $n_{j}(x, t) \rightarrow n(x, t)$ pointwise (cf. [4]). It follows that $n(x, t)$ satisfies (3.4) and consequently (3.3), or (3.2), holds.

THEOREM 3.2. If (1.1), (2.2) hold and

$$
n_{0}(x, c)=0 \text { for } x \geq 0, c>c_{*}\left(c_{*}>0\right) \text {, }
$$

then

$$
n(x, c, t)=0 \text { for } x \geq 0, c \geq c_{*} t \geq 0 \text {. }
$$

Proof. If $c>c_{*}$ then in the first integral on the right-hand side of (1.4)

$$
\text { either } \gamma>c_{*} \text { or else } \frac{x c-\xi \gamma}{x-\xi}>c_{*} \text {. }
$$

Assuming that $K$ has compact support and using (2.3), we conclude that the integral is bounded by $C N(t)$, where

$$
N(t)=\sup _{x \geq 0, c \geq c_{*}} n(x, c, t)
$$

If $B$ has compact support then the second integral on the right-hand side of (1.4) is bounded by

$$
C \sup _{\xi>x} n(\xi, c, t), \quad \text { or by } C N(t)
$$


From (1.4) it then follows that

$$
\frac{d n(x, c, t)}{d t} \leq C N(t) .
$$

If $x \geq 0, c>c_{*}$, so that

$$
N(t) \leq N(0)+C \int_{0}^{t} N\left(t^{\prime}\right) d t^{\prime} .
$$

Since $N(0)=0$, the assertion (3.6) follows.

Having proved (3.6) in the case where $K$ and $B$ have compact support, we can now establish the same result for general $K, B$ by approximation (cf. the proof of Theorem 3.1).

For convenience we collect all the assumptions made so far on $n_{0}(x, c)$ and $n_{0}(x)$ :

$$
\left\{\begin{array}{l}
n_{0}(x) \text { and } n_{0}(x, c) \text { are continuous functions for } x \geq 0, c \geq 0 \\
0 \leq n_{0}(x) \leq A_{0}, \quad \int_{0}^{\infty}(1+x) n_{0}(x) d x<\infty \\
0 \leq n_{0}(x, c) \leq A_{1}, \quad \int_{0}^{\infty} n_{0}(x, c) d c=n_{0}(x) \\
n_{0}(x, c)=0 \text { if } c \geq c_{*}\left(c_{*}>0\right) .
\end{array}\right.
$$

Corollary 3.3. If (1.1) and (3.7) are satisfied then (3.2) and (3.6) hold.

4. A formula for $\boldsymbol{d} \boldsymbol{S} / \boldsymbol{d} \boldsymbol{t}$. Let $f(x, c)$ be any function satisfying

$$
|f(x, c)| \leq C(1+x) \text { if } x \geq 0, c \leq c_{*}
$$

and arbitrary otherwise. By (2.6) and Corollary 3.3 it follows that the integral

$$
S(t)=\int_{0}^{\infty} \int_{0}^{\infty} f(x, c) n(x, c, t) d x d c
$$

exists for all $t \geq 0$.

THEOREM 4.1. If (1.1), (3.7) hold and $f$ satisfies (4.1), then

$$
\begin{aligned}
\frac{d S(t)}{d t}= & \frac{1}{2} \int_{0}^{\infty} d x \int_{0}^{\infty} d \xi \int_{0}^{\infty} d c \int_{0}^{\infty} d \gamma K(x, \xi) n(x, c, t) n(\xi, \gamma, t) \\
& \times\left[f\left(x+\xi, \frac{x c+\xi \gamma}{x+\xi}\right)-f(x, c)-f(\xi, \gamma)\right] \\
+ & \int_{0}^{\infty} d x \int_{0}^{\infty} d c \int_{x}^{\infty} B(\xi, x) n(\xi, c, t)\left[f(x, c)-\frac{x}{\xi} f(\xi, c)\right] d \xi .
\end{aligned}
$$

Note that, by (2.6) and Corollary 3.3, all the integrals on the right-hand side of (4.3) are absolutely convergent. 
Proof. Formally

$$
\frac{d S(t)}{d t}=\int_{0}^{\infty} \int_{0}^{\infty} f(x, c) \frac{d n(x, c, t)}{d t} d x d c
$$

By substituting $d n / d t$ from (1.4) and using (2.6), (3.2), (4.1), we find that each of the resulting four integrals is absolutely convergent.

A standard argument then shows that $S(t)$ is differentiable and $d S / d t$ is given by (4.4).

We can next write

$$
\frac{d S}{d t}=I_{1}+I_{2}
$$

where

$$
\begin{aligned}
I_{1}= & \int_{0}^{\infty} d c\left[\int_{0}^{\infty} f(x, c) d x \int_{x}^{\infty} B(\xi, x) n(\xi, c, t) d \xi\right. \\
& \left.-\int_{0}^{\infty} f(x, c) n(x, c, t) d x \int_{0}^{x} \frac{\xi}{x} B(x, \xi) d \xi\right], \\
I_{2}= & \frac{1}{2} \int_{0}^{\infty} d x \int_{0}^{\infty} f(x, c) d c \int_{0}^{x} \int_{0}^{\infty} K(x-\xi, \xi) n\left(x-\xi, \frac{x c-\xi \gamma}{x-\xi}, t\right) n(\xi, \gamma, t) \frac{x}{x-\xi} d \gamma d \xi \\
& \quad-\int_{0}^{\infty} d c \int_{0}^{\infty} d x \int_{0}^{\infty} n(x, c, t) K(x, \xi) n(\xi, \gamma, t) f(x, c) d \gamma d \xi \\
= & I_{21}-I_{22} .
\end{aligned}
$$

To evaluate $I_{1}$ we change the notation of the variables $x \rightarrow \xi$ and $\xi \rightarrow x$ in the second integral in $[\cdots]$ to get

$$
\int_{0}^{\infty} d \xi \int_{0}^{\xi} \frac{x}{\xi} B(\xi, c) f(\xi, c) n(\xi, c, t) d x
$$

This, in turn, is equal, by changing the order of integration, to

$$
\int_{0}^{\infty} d x \int_{x}^{\infty} \frac{x}{\xi} B(\xi, x) f(\xi, c) n(\xi, c, t) d \xi
$$

Hence

$$
I_{1}=\int_{0}^{\infty} d c \int_{0}^{\infty} d x \int_{x}^{\infty} B(\xi, x)\left[f(x, c)-\frac{x}{\xi} f(\xi, c)\right] n(\xi, c, t) d \xi
$$

Next we turn to $I_{21}$ and change variables $x \rightarrow x^{\prime}$ where $x-\xi=x^{\prime}$ (for each fixed $\xi$ ) and $c \rightarrow c^{\prime}$ by

$$
c=\frac{x^{\prime} c^{\prime}+\xi \gamma}{x^{\prime}+\xi}, \text { or } \frac{x c-\xi \gamma}{x-\xi}=c^{\prime}
$$


We get

$$
I_{21}=\frac{1}{2} \int_{0}^{\infty} d x^{\prime} \int_{0}^{\infty} d \xi \int_{0}^{\infty} d c^{\prime} \int_{0}^{\infty} d \gamma K\left(x^{\prime}, \xi\right) n\left(x^{\prime}, c, t\right) n(\xi, \gamma, t) f\left(\xi+x^{\prime}, \frac{x^{\prime} c^{\prime}+\xi \gamma}{x^{\prime}+\xi}\right) .
$$

We shall use the relations

$$
\begin{aligned}
\int_{0}^{\infty} d c^{\prime} \int_{0}^{\infty} d \gamma \Phi & =\int_{0}^{\infty} d c^{\prime} \int_{0}^{c^{\prime}} d \gamma \Phi+\int_{0}^{\infty} d c^{\prime} \int_{c^{\prime}}^{\infty} d \gamma \Phi \\
& =\int_{0}^{\infty} d c^{\prime} \int_{0}^{c^{\prime}} d \gamma \Phi+\int_{0}^{\infty} d \gamma \int_{0}^{\gamma} d c^{\prime} \Phi
\end{aligned}
$$

which is valid with any integrand $\Phi$. Since after integration, in $I_{21}$, with respect to $x^{\prime}$ and $\xi$ we get an expression $\Psi\left(\gamma, c^{\prime}\right)$ which is symmetric in $\gamma, c^{\prime}$, we can write

$$
\int_{0}^{\infty} d \gamma \int_{0}^{\gamma} d c^{\prime} \Psi\left(\gamma, c^{\prime}\right)=\int_{0}^{\infty} d c^{\prime} \int_{0}^{c^{\prime}} d \gamma \Psi\left(\gamma, c^{\prime}\right)
$$

Hence for the purpose of calculating $I_{21}$ we may replace the right-hand side of (4.7) by

$$
2 \int_{0}^{\infty} d c^{\prime} \int_{0}^{c^{\prime}} d \gamma \Phi
$$

Consequently

$$
I_{21}=\int_{0}^{\infty} d x^{\prime} \int_{0}^{\infty} d \xi \int_{0}^{\infty} d c^{\prime} \int_{0}^{c^{\prime}} d \gamma K\left(x^{\prime}, \xi\right) n\left(x^{\prime}, c^{\prime}, t\right) n(\xi, \gamma, t) f\left(x^{\prime}+\xi, \frac{x^{\prime} c^{\prime}+\xi \gamma}{x^{\prime}+\xi}, t\right) .
$$

Next we can write

$$
\begin{aligned}
I_{22} & =\int_{0}^{\infty} d x \int_{0}^{\infty} d \xi \int_{0}^{\infty} d c \int_{0}^{c} d \gamma K(x, \xi) n(x, c, t) n(\xi, \gamma, t) f(x, c) \\
& +\int_{0}^{\infty} d x \int_{0}^{\infty} d \xi \int_{0}^{\infty} d c \int_{0}^{\infty} d \gamma K(x, \xi) n(x, c, t) n(\xi, \gamma, t) f(x, c) \\
& =L_{1}+L_{2}
\end{aligned}
$$

In $L_{2}$ we write

$$
\int_{0}^{\infty} d c \int_{c}^{\infty} d \gamma=\int_{0}^{\infty} d \gamma \int_{0}^{\gamma} d c
$$

to get

$$
L_{2}=\int_{0}^{\infty} d x \int_{0}^{\infty} d \xi \int_{0}^{\infty} d \gamma \int_{0}^{\gamma} d c K(x, \xi) n(x, c, t) n(\xi, \gamma, t) f(x, c)
$$


Changing the notation of variables $\gamma \leftrightarrow c$ and $x \leftrightarrow \xi$ we find, using the symmetry of $K(x, \xi)$, that

$$
L_{2}=\int_{0}^{\infty} d \xi \int_{0}^{\infty} d x \int_{0}^{\infty} d c \int_{0}^{c} d \gamma K(x, \xi) n(\xi, \gamma, t) n(x, c, t) f(\xi, c)
$$

We substitute this expression into (4.9) and, upon renaming $x^{\prime}, c^{\prime}$ in (4.8) by $x, c$, obtain

$$
\begin{aligned}
I_{2}=I_{21}-I_{22} & =\int_{0}^{\infty} d x \int_{0}^{\infty} d \xi \int_{0}^{\infty} d c \int_{0}^{c} d \gamma K(x, \xi) n(x, c, t) n(\xi, \gamma, t) \\
& \times\left[f\left(x+\xi, \frac{x c+\xi \gamma}{x+\xi}\right)-f(x, c)-f(\xi, \gamma)\right] .
\end{aligned}
$$

Next we write in $I_{2}$

$$
\cdots \int_{0}^{\infty} d c \int_{0}^{c} d \gamma \text { as } \quad \cdots \int_{0}^{\infty} d \gamma \int_{\gamma}^{\infty} d c
$$

and change notation $c \leftrightarrow \gamma, x \leftrightarrow \xi$. We find that $I_{2}$ is equal to a similar integral in which

$$
\int_{0}^{\infty} d c \int_{0}^{c} d \gamma \text { is replaced by } \int_{0}^{\infty} d c \int_{c}^{\infty} d \gamma
$$

(here we again use the symmetry of $K(x, \xi)$ ). It follows that $I_{2}$ is equal to $\frac{1}{2}$ times the first integral on the right-hand side of (4.3). Recalling also (4.6), (4.5), the assertion (4.3) follows.

Formula (4.3) will play a key role in the subsequent sections. Note that the choice $f=x$ gives the conservation law (3.2) which was already proved. On the other hand the choice $f=x c$ gives a new conservation law:

$$
\int_{0}^{\infty} \int_{0}^{\infty} x c n(x, c, t) d x c d c=\int_{0}^{\infty} \int_{0}^{\infty} x c n_{0}(x, c) d x d c \text { for all } t \geq 0 .
$$

COROLlaRY 4.2. If $g(c)$ is a convex function then

$$
\frac{d}{d t} \int_{0}^{\infty} \int_{0}^{\infty} g(c) x n(x, c, t) d x d c \leq 0
$$

Indeed, the second integral on the right-hand side of (4.3) is zero whereas the first integral is $\leq 0$

Corollary 4.3. If $n_{0}(x, c)=0$ for $0 \leq c \leq \tilde{c}, x \geq 0$ then $n(x, c, t)=0$ for $0 \leq c \leq \tilde{c}$, $x \geq 0, t \geq 0$.

Indeed, taking $g(c)$ to be the convex function $(\tilde{c}-c)^{+}$we conclude that

$$
\int_{0}^{\infty} \int_{0}^{\tilde{c}}(\tilde{c}-c)^{+} x n(x, c, t) d x d c \leq \int_{0}^{\infty} \int_{0}^{\tilde{c}}(\tilde{c}-c)^{+} x n_{0}(x, c) d x d c=0
$$


and the assertion follows.

The proof of Theorem 4.1 can also be applied (or, rather, specialized) to functions of the form

$$
\tilde{S}(t)=\int_{0}^{\infty} f(x) n(x, t) d x
$$

We then get:

THEOREM 4.4. If (1.1), (3.7) hold and $f(x)$ satisfies

$$
|f(x)| \leq C(1+x)
$$

then

$$
\begin{aligned}
\frac{d \tilde{S}(t)}{d t} & =\frac{1}{2} \int_{0}^{\infty} \int_{0}^{\infty} K(x, \xi) n(x, t) n(\xi, t)[f(x+\xi)-f(x)-f(\xi)] d x d \xi \\
& +\int_{0}^{\infty} d x \int_{x}^{\infty} B(\xi, x) n(\xi, t)\left[f(x)-\frac{x}{\xi} f(\xi)\right] d \xi
\end{aligned}
$$

5. $\boldsymbol{D}(\boldsymbol{t}) \rightarrow \mathbf{0}$ if $\boldsymbol{t} \rightarrow \infty$. In this section we impose additional mild assumptions on $K, B$ and $n_{0}$ :

$$
\int_{0}^{\infty} x^{2} n_{0}(x) d x<\infty
$$

and

(a) $\quad K(x, \xi) \leq C(x+\xi)$;

(b) $\partial_{x} K(x, \xi) \geq-C$;

(c) $\quad \exists$ a positive constant $\gamma_{1}$ such that, for any $N \gg 1$, $K(x, \xi) \geq \frac{\gamma_{1}}{N}(x+\xi)$ if $0 \leq x, \xi \leq N$

(d) $\int_{0}^{\xi} B(\xi, x) x(\xi-x) d x \geq \gamma_{2} \xi^{2} \forall \xi>0$, for some $\gamma_{2}>0$.

Remark 1.1 indicates that condition $(5.2)(\mathrm{d})$ is quite natural. Note also that $(5.2)(\mathrm{c})$ is satisfied if

$$
K(x, \xi) \geq \begin{cases}\delta(x+\xi) & \text { for } x+\xi<1 \\ \delta & \text { for } x+\xi>1\end{cases}
$$

where $\delta$ is a small positive constant.

Set

$$
\begin{aligned}
M_{0} & =\int_{0}^{\infty} \int_{0}^{\infty} x n_{0}(x, c) d x d c, \quad M_{1}=\int_{0}^{\infty} \int_{0}^{\infty} x c n_{0}(x, c) d x d c \\
c_{\infty} & =\frac{M_{1}}{M_{0}}
\end{aligned}
$$


In this section we prove:

ThEOREM 5.1. If (1.1), (3.7) and (5.1), (5.2) hold, then the function

$$
D(t) \equiv \int_{0}^{\infty} \int_{0}^{\infty} x\left(c-c_{\infty}\right)^{2} n(x, c, t) d x d c
$$

converges to zero as $t \rightarrow \infty$.

We need several lemmas.

LEMMA 5.2. There exists a constant $C$ independent of $t$ such that

$$
\int_{0}^{\infty} x^{2} n(x, t) d x \leq C \quad \forall t>0 .
$$

Proof. Consider first the case where

$$
K, B \text { and } n_{0} \text { have compact support. }
$$

Then, for any $T>0$,

$$
\tilde{S}(t) \equiv \int_{0}^{\infty} x^{2} n(x, t) d x \leq C(T)<\infty \text { if } 0 \leq t \leq T
$$

and we may apply (4.13) with $f(x)=x^{2}$ (The proof is the same as that of Theorem 4.4.). We get

$$
\begin{aligned}
\frac{d \tilde{S}}{d t} & \leq C \int_{0}^{\infty} \int_{0}^{\infty} n(x, t) n(\xi, t) x \xi d x d \xi \\
& +\int_{0}^{\infty} n(\xi, t) d \xi \int_{0}^{\xi} B(\xi, x)\left(x^{2}-x \xi\right) d x
\end{aligned}
$$

since $K(x, \xi) \leq C$. Using Theorem 3.1 and (5.2) (d) we obtain

$$
\frac{d \tilde{S}}{d t} \leq C-\gamma_{2} \int_{0}^{\infty} \xi^{2} n(\xi, t) d \xi=C-\gamma_{2} \tilde{S}(t)
$$

Consequently

$$
\tilde{S}(t) \leq \tilde{S}(0) e^{-\gamma_{2} t}+\frac{C}{\gamma_{2}} .
$$

and (5.6) follows with a constant which is independent of the assumptions in (5.7). Approximating general $K, B, n_{0}$ by functions with compact support and applying (5.8) to the corresponding solutions, we obtain, in the limit, the assertion (5.6).

Introduce the function

$$
S_{2}(t)=\int_{0}^{\infty} \int_{0}^{\infty} x c^{2} n(x, c, t) d x d c
$$


Then

$$
D(t)=S_{2}(t)-2 c_{\infty} M_{1}+C_{\infty}^{2} M_{0}
$$

LEMMA 5.3. The function $S_{2}(t)$ satisfies:

$$
\begin{aligned}
\frac{d S_{2}(t)}{d t} & \leq 0 \\
\frac{d^{2} S_{2}(t)}{d t^{2}} & \leq C
\end{aligned}
$$

for all $t>0$, and

$$
\frac{d S_{2}(t)}{d t} \rightarrow 0 \text { if } t \rightarrow \infty
$$

Proof. Using (4.3) with $f(x, c)=x c^{2}$ and noting that

$$
f\left(x+\xi, \frac{x c+\xi \gamma}{x+\xi}\right)-f(x, c)-f(\xi, \gamma)=-\frac{x \xi}{x+\xi}(c-\gamma)^{2}
$$

we get

$$
\frac{d S_{2}}{d t}=-\frac{1}{2} \int_{0}^{\infty} d x \int_{0}^{\infty} d \xi \int_{0}^{\infty} d c \int_{0}^{\infty} d \gamma \frac{K(x, \xi)}{x+\xi} x \xi(c-\gamma)^{2} n(x, c, t) n(\xi, \gamma, t),
$$

so that $d S_{2} / d t \leq 0$.

To prove (5.12) we want to apply Theorem 4.1 with

$$
f(x, c)=\frac{K(x, \xi)}{x+\xi} x(c-\gamma)^{2} ; \quad \xi, \gamma \text { are parameters. }
$$

for simplicity we first take $B=0$. Then

$$
\begin{aligned}
\frac{d^{2} S_{2}}{d t^{2}}= & -\frac{1}{2} \int_{0}^{\infty} d x \int_{0}^{\infty} d \xi \int_{0}^{\infty} d c \int_{0}^{\infty} d \gamma \xi n(\xi, \gamma, t) \int_{0}^{\infty} d \zeta \int_{0}^{\infty} d \sigma K(x, \xi) n(x, c, t) n(\xi, \sigma, t) \\
\times & {\left[\frac{K(x+\zeta, \xi)}{x+\zeta+\xi}(x+\zeta)\left(\frac{x c+\zeta \sigma}{x+\zeta}-\gamma\right)^{2}-\frac{K(x, \xi)}{x+\xi} x(c-\gamma)^{2}\right.} \\
& \left.-\frac{K(\zeta, \xi)}{\zeta+\xi} \zeta(\sigma-\gamma)^{2}\right] .
\end{aligned}
$$

Using

$$
\frac{x c+\zeta \sigma}{x+\zeta}-\gamma=\frac{x(c-\gamma)+\zeta(\sigma-\gamma)}{x+\zeta}
$$

and a calculation similar to (5.14), we find that the expression in brackets is equal to

$$
\begin{aligned}
\frac{K(x+\zeta, \xi)}{x+\zeta+\xi}\left(\frac{-x \zeta(c-\sigma)^{2}}{x+\zeta}\right)+ & +\left(\frac{K(x+\zeta, \xi)}{x+\zeta+\xi}-\frac{K(x, \xi)}{x+\xi}\right) x(c-\gamma)^{2} \\
+ & \left(\frac{K(x+\zeta, \xi)}{x+\zeta+\xi}-\frac{K(\zeta, \xi)}{\zeta+\xi}\right) \zeta(\sigma-\gamma)^{2} .
\end{aligned}
$$


By $(5.2)(\mathrm{a}), K(x, \zeta)$ times the first term is bounded by $c x \zeta$, and, by $(5.2)(\mathrm{a})$ and $(5.2)(\mathrm{b})$, $K(x, \zeta)$ times each of the remaining two terms is bounded from below by $-C x \zeta$.

It follows that $d^{2} S_{2} / d t^{2}$ is bounded from above by

$$
C\left(\int_{0}^{\infty} \int_{0}^{\infty} x n(x, c, t) d x d t\right)^{3} \leq \text { const. }
$$

If $B \neq 0$ then we have to estimate the corresponding positive contribution to $d^{2} S_{2} / d t^{2}$ :

$$
\frac{1}{2} \int_{0}^{\infty} d x \int_{0}^{\infty} d \xi \int_{0}^{\infty} d c \int_{0}^{\infty} d \gamma \xi n(\xi, \gamma, t) f(x, c) n(x, c, t) \int_{0}^{x} \frac{\zeta}{x} B(x, \zeta) d \zeta
$$

where $f(x, c)$ is defined as in (5.16). Since $K(x, \xi) \leq C(x+\xi)$, we have $f(x) \leq C x$. Recalling also the last condition in (1.1) we find that the expression in (5.17) is bounded by

$$
C\left(\int_{0}^{\infty} \int_{0}^{\infty} x n(x, c, t) d x d c\right)^{2} \leq \text { const. }
$$

This completes the proof of (5.12).

It remains to prove (5.13). If the assertion is not true then there is a sequence $t_{n} \rightarrow \infty$ such that

$$
0 \geq S_{2}^{\prime}\left(t_{n}\right) \rightarrow-\alpha<0 \text { if } n \rightarrow \infty
$$

Using (5.12) we get

$$
S_{2}\left(t_{n}+\epsilon\right)-S_{2}\left(t_{n}\right)=\epsilon S_{s}^{\prime}\left(t_{n}\right)+\frac{\epsilon^{2}}{2} S_{2}^{\prime \prime}\left(\tilde{t}_{n}\right) \leq-\frac{\alpha \epsilon}{2}
$$

if $\epsilon$ is positive and small enough. It follows that we have $f(x, c) \leq C x$.

$$
S_{2}\left(t_{n}+\epsilon\right) \leq S_{2}\left(t_{1}\right)-\frac{n \epsilon}{2}<0
$$

if $n$ is sufficiently large, a contradiction.

For any $N \gg 1$ introduces the quantities

$$
\begin{aligned}
M_{0, N} & =\int_{0}^{N} d x \int_{0}^{\infty} x n(x, c, t) d c \\
M_{1, N}(x) & =\int_{0}^{N} d x \int_{0}^{\infty} c x n(x, c, t) d c
\end{aligned}
$$

and

$$
S_{2, N}(t)=\int_{0}^{n} d x \int_{0}^{\infty} c^{2} x n(x, c, t) d c
$$


By Lemma 5.2,

$$
\left\{\begin{array}{l}
\left|S_{2}(t)-S_{2, N}(t)\right| \leq \frac{C}{N} \text { for all } t \geq 0 \\
\left|M_{0, N}-M_{0}\right|+\left|M_{1, N}-M_{1}\right| \leq \frac{C}{N}
\end{array}\right.
$$

LEMma 5.4. There holds:

$$
\int_{0}^{\infty} d x \int_{0}^{\infty} d c \int_{0}^{\infty} d \xi \int_{0}^{\infty} d \gamma x \xi(c-\gamma)^{2} n(x, c, t) n(\xi, \gamma, t)=2\left(S(t) M_{0}-M_{1}^{2}\right)
$$

and, in particular,

$$
S(t) M_{0} \geq M_{1}^{2}
$$

The proof is obvious.

Proof of Theorem 5.1. by (5.15)

$$
\frac{d S_{2}(t)}{d t}=-\frac{1}{2} \iiint \int_{x<N, \xi<N} d x d c d \xi d \gamma \frac{K(x, \xi)}{x+\xi} x \xi(c-\gamma)^{2} n(x, c, t) n(\xi, \gamma, t)+J_{N}
$$

where, since $K(x, \xi) \leq C$,

$$
\left|J_{N}\right| \leq C \iiint \int_{\xi>N} d x d c d \xi d \gamma x n(x, c, t) n(\xi, \gamma, t) \leq \frac{C}{N^{2}}
$$

the last inequality follows by Lemma 5.2. Using (5.2)(c) we conclude that

$$
\frac{d S_{2}}{d t} \leq-\frac{\gamma_{1}}{N} \iiint \int_{x<N, \xi<N} d x d c d \xi d \gamma x \xi(c-\gamma)^{2} n(x, c, t) n(\xi, \gamma, t)+\frac{C}{N^{2}}
$$

Evaluating the last integral as in (5.19), we get

$$
\frac{d S_{2}}{d t} \leq-\frac{2 \gamma_{1}}{N}\left(S_{2, n}(t) M_{0, N}-M_{1, N}^{2}\right)+\frac{C}{N^{2}}
$$

so that

$$
S_{2, N}(t) M_{0, N}-M_{1, N}^{2} \leq-\frac{N}{2 \gamma_{1}} S_{2}^{\prime}(t)+\frac{C}{N}
$$

Using (5.18) we deduce that

$$
S_{2}(t) M_{0}-M_{1}^{2} \leq-\frac{N}{2 \gamma_{1}} S_{2}^{\prime}(t)+\frac{C}{N}
$$

Taking $t \rightarrow \infty$ and using (5.13) we find that

$$
\varlimsup_{t \rightarrow \infty}\left(S(t) M_{0}-M_{1}^{2}\right) \leq \frac{C}{N}
$$


Taking $N \rightarrow \infty$ and recalling (5.20), we find that

$$
S(t) M_{0}-M_{1}^{2} \rightarrow 0 \quad \text { if } t \rightarrow \infty .
$$

Consequently, as $t \rightarrow \infty$,

$$
D(t) \rightarrow \frac{M_{1}^{2}}{M_{0}}-2 C_{\infty} M_{1}+C_{\infty}^{2} M_{0}=0, \quad \text { by }(5.10) \text { and }(5.4),
$$

and the proof of Theorem 5.1 is complete

Remark 5.5. Dubovskii and Stewart [4] proved existence and uniqueness for (1.2), (1.3) also in case $K(x, \xi)$ is unbounded, provided

$$
\begin{aligned}
K(x, \xi) & \leq C(x+\xi) \\
n_{0}(x) & \leq C e^{-\lambda x} \quad(\lambda>0)
\end{aligned}
$$

The solution is exponentially decreasing, and uniqueness is in the class of exponentially decreasing functions. Their result can be extended to (1.4), (1.5). Suppose now that $K$ also satisfies:

$$
K(x, \xi) \geq \gamma_{3}(x+\xi), \quad \gamma_{3}>0
$$

Then the proof of Theorem 5.1 remains valid without actually splitting integrals by $x \geq N$ or $\xi_{\gtrless N}$. Thus we get, in this case,

$$
\frac{d S_{2}}{d t} \leq-\gamma_{3}\left(S_{2}(t) M_{0}-M_{1}^{2}\right)
$$

The non-negative function

$$
T(t)=S_{2}(t)-\frac{M_{1}^{2}}{M_{0}}
$$

then satisfies

$$
\frac{d T}{d t}+2 \gamma_{3} T \leq 0
$$

so that

$$
0 \leq T(t) \leq T(0) e^{-2 \gamma_{2} t}
$$

We conclude that, as $t \rightarrow \infty$,

$$
D(t) \rightarrow 0 \text { exponentially fast. }
$$

Note that in this case we do not require the assumption $(5.2)(\mathrm{d})$. 


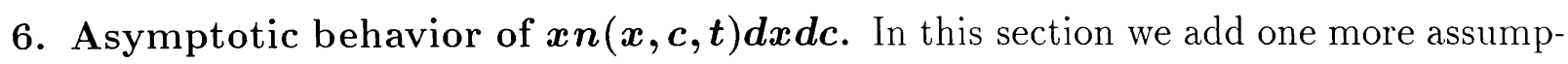
tion on $K, B, n_{0}$ :

$$
K(x, \xi) \leq \eta(x+\xi) \text { where } 2 M_{0} \eta<\gamma_{2}, \quad \gamma_{2} \text { as in }(5.2)(\mathrm{d}) .
$$

Theorem 6.1. Let (1.1), (3.7), (5.1), (5.2) and (6.1) hold. Then there exists a nonnegative measure $d L(x)$ such that

$$
\lim _{t \rightarrow \infty} \int_{0}^{\infty} \int_{0}^{\infty} g(x, c) x n(x, c, t) d x d c=\int_{0}^{\infty} g\left(x, c_{\infty}\right) d L(x)
$$

for any bounded continuous function $g(x)$.

Introducing the measures

$$
d \mu_{t}(x, c)=x n(x, c, t) d x d c
$$

we can restate $(6.2)$ in the form:

$$
d \mu_{t}(x, c) \rightarrow \delta\left(c-c_{\infty}\right) d L(x) \text { as } t \rightarrow \infty, \text { in the sense of weak convergence, }
$$

where $\delta(y)$ is the Dirac measure.

The proof requires several lemmas.

LEMMA 6.2. Under the assumptions of Theorem 6.1.

$$
\lim _{t \rightarrow \infty} \int_{0}^{\infty} g(x) x n(x, t) d x \text { exists }
$$

for any bounded continuous function $g(x)$.

Proof. Consider first the function

$$
S_{0}(t)=\int_{0}^{\infty} x^{2} n(x, t) d x
$$

By $(4.13)$

$$
\begin{aligned}
\frac{d S_{0}}{d t} & =\int_{0}^{\infty} \int_{0}^{\infty} K(x, \xi) n(x, t) n(\xi, t) x \xi d x d \xi \\
& +\int_{0}^{\infty} d x \int_{x}^{\infty} B(\xi, x) n(\xi, t)\left(x^{2}-x \xi\right) d \xi \\
\leq & \eta \int_{0}^{\infty} \int_{0}^{\infty}(x+\xi) n(x, t) n(\xi, t) x \xi d x d \xi \\
\leq & \quad+\int_{0}^{\infty} n(\xi, t) d \xi \int_{0}^{\infty} B(\xi, x)\left(x^{2}-x \xi\right) d x \\
\leq &
\end{aligned}
$$


so that

$$
\frac{d S_{0}}{d t} \leq-\delta S_{0}(t), \quad \delta=\gamma_{2}-2 \eta M_{0}>0
$$

Suppose now that $g(x)$ is a uniformly Lipschitz continuous function and write

$$
\begin{aligned}
\int_{0}^{\infty} g(x) x n(x, t) d x & =\int_{0}^{\infty}\left(g(x) x+N x^{2}\right) n(x, t) d x \\
& -N \int_{0}^{\infty} x^{2} n(x, t) d x \equiv \tilde{S}_{1}(t)-N S_{0}(t) .
\end{aligned}
$$

By $(6.5), S_{0}(t)$ is monotone decreasing, and hence

$$
\lim _{t \rightarrow \infty} S_{0}(t) \text { exists. }
$$

Next, by Theorem 4.4 with $f(x)=x g(x)$,

$$
\begin{aligned}
& \frac{d}{d t} \int_{0}^{\infty} g(x) x n(x, t) d x \\
& \leq \frac{1}{2} \int_{0}^{\infty} d x \int_{0}^{\infty} d \xi \eta(x+\xi) n(x, t) n(\xi, t) x \xi \\
& \quad \times\left[\frac{g(x+\xi)-g(x)}{\xi}+\frac{g(x+\xi)-g(\xi)}{x}\right] \\
& +\int_{0}^{\infty} d x \int_{x}^{\infty} B(\xi, x) n(\xi, t) x[g(x)-g(\xi)] d \xi .
\end{aligned}
$$

The first integral on the right-hand side is bounded by

$$
C \int_{0}^{\infty} x^{2} n(x, t) d x \cdot \int_{0}^{\infty} \xi n(\xi, t) d \xi
$$

and the second integral is bounded by

$$
\int_{0}^{\infty} n(\xi, t) d \xi \int_{0}^{\xi} B(\xi, x) x C|x-\xi| d x \leq C \int_{0}^{\infty} \xi^{2} n(\xi, t) d \xi .
$$

Recalling (6.5), it follows that

$$
\frac{d \tilde{S}_{1}}{d t} \leq-\delta N S_{0}(t)+C_{0} S_{0}(t)<0 \quad \text { if } N>\frac{C_{0}}{\delta}
$$

and consequently

$$
\lim _{t \rightarrow \infty} \tilde{S}_{1}(t) \quad \text { exists. }
$$


Combining this with (6.7) we deduce from (6.6) that (6.4) holds for any uniformly Lipschitz continuous function $g(x)$.

Consider next the case where $g(x)$ is any bounded continuous function and let $g_{\epsilon}$ be a mollifier of $g$ :

$$
\left|g_{\epsilon}(x)-g(x)\right|<\epsilon \text { and }\left|g_{\epsilon}^{\prime}(x)\right| \leq \frac{C}{\epsilon} \quad \text { for all } x \geq 0
$$

Write

$$
\begin{aligned}
\int_{0}^{\infty} g(x) x n(x, t) d x & =\int_{0}^{\infty} g_{\epsilon}(x) x n(x, t) d x \\
& +\int_{0}^{\infty}\left(g(x)-g_{\epsilon}(x)\right) x n(x, t) d x \equiv T_{1}(t)+T_{2}(t) .
\end{aligned}
$$

Clearly

$$
\left|T_{2}(t)\right|<\epsilon
$$

By what was already proved above

$$
A_{\epsilon} \equiv \lim _{t \rightarrow \infty} T_{1}(t) \text { exists. }
$$

Hence

$$
\lim _{t \rightarrow \infty}\left|\int_{0}^{\infty} g(x) x n(x, t) d x-A_{\epsilon}\right| \leq \epsilon .
$$

Since the $A_{\epsilon}$ are uniformly bounded, we can take a sequence $\epsilon=\epsilon_{m} \rightarrow 0$ such that $A_{\epsilon_{m}} \rightarrow A$, and obtain

$$
\varlimsup_{t \rightarrow \infty}\left|\int_{0}^{\infty} g(x) x n(x, t) d x-A\right|=0 .
$$

This completes the proof of Lemma 6.2.

LEMMA 6.3. Under the assumptions of Theorem 6.1,

$$
\lim _{t \rightarrow \infty} \int_{0}^{\infty} \int_{0}^{\infty} g(x, c) x n(x, c, t) d x d c \text { exists }
$$

for any bounded continuous function $g(x, c)$.

The proof is similar to the proof of Lemma 6.2 and will be omitted. Note that here we work (cf.(6.6)) with

$$
\left(g(x, c)+N x^{2}\right) \text { and }-N x^{2} .
$$

The limit in (6.8) is a bounded linear functional $\Phi$ on the space of bounded continuous functions $g$ equipped with the $L^{\infty}$ norm. By [6, pp. 261-262] we can write

$$
\begin{aligned}
\lim _{t \rightarrow \infty} \int_{0}^{\infty} \int_{0}^{\infty} g(x, c) x n(x, c, t) d x d c \\
\quad=\iint g(x, c) d N(x, c) \equiv \Phi(g)
\end{aligned}
$$


where $d N$ is a bounded finitely additive regular set function. It will be also useful to view the right-hand side of (6.9) as a distribution, and use the properties

$$
\begin{aligned}
|\Phi(g)| & \leq C|g|_{L^{\infty}} \\
\Phi(g) & \geq 0 \text { if } g \geq 0 .
\end{aligned}
$$

From Theorem 5.1 it follows that

$$
\iint\left(c-c_{\infty}\right)^{2} d N(x, c)=0
$$

and therefore also

$$
\iint g(x, c)\left(c-c_{\infty}\right)^{2} d N(x, c)=0
$$

for any bounded continuous function $g(x, c)$.

If $g(x, c)$ vanishes in a neighborhood of the line $\left\{c=c_{\infty}\right\}$ then we can apply $(6.11)$ to the function

$$
\tilde{g}(x, c)=\left\{\begin{array}{l}
\frac{g(x, c)}{\left(c-c_{\infty}\right)^{2}} \text { on suppg } \\
0 \text { elsewhere }
\end{array}\right.
$$

and conclude that

$$
\Phi(g)=\iint \tilde{g}(x, c)\left(c-c_{\infty}\right)^{2} d N(x, c)=0 .
$$

It follows that the support of the distribution $\Phi$ lies in the set $\left\{c=c_{\infty}\right\}$. By a standard result in the theory of distributions (e.g. [7, p. 69, Theorem 31], $\Phi$ must then have the form

$$
\Phi(g)=\sum_{0 \leq i, j \leq l} \frac{\partial^{i+j}}{\partial c^{i} \partial x^{j}} \int g\left(x, c_{\infty}\right) d k_{i j}(x)
$$

where the derivatives $\partial^{i+j} / \partial c^{i} \partial x^{j}$ are taken in the sense of distributions, and $d k_{i j}$ are measures. In view of $(6.10)$ we must have $l=0$ and, furthermore, $d k_{00}$ is a non-negative measure. This completes the proof of Theorem 6.1 .

The equilibrium equation (1.1) is

$$
\begin{aligned}
& \frac{1}{2} \int_{0}^{\infty} K(x-\xi, \xi) n_{\infty}(x-\xi) n_{\infty}(\xi) d \xi+\int_{x}^{\infty} B(\xi, x) n_{\infty}(\xi) d \xi \\
& \quad-n_{\infty}(x) \int_{0}^{\infty} K(x, \xi) n_{\infty}(\xi) d \xi-n_{\infty}(x) \int_{0}^{x} \frac{\xi}{x} B(x, \xi) d \xi=0 .
\end{aligned}
$$

If $n_{\infty}(x)$ is a bounded, non-negative, $L^{1}(0, \infty)$-solution of $(6.12)$, then the measure $d L(x)=$ $x n_{\infty}(x) d x$ satisfies:

$$
\begin{aligned}
& \frac{1}{2} \int_{0}^{\infty} d L(\xi) \int_{0}^{\infty} d L(x) K(x, \xi)\left[\frac{g(x+\xi)-g(x)}{\xi}+\frac{g(x+\xi)-g(\xi)}{x}\right] \\
& \quad+\int_{0}^{\infty} d L(\xi) \int_{0}^{\xi} B(\xi, x) \frac{x}{\xi}(g(x)-g(\xi)) d x=0
\end{aligned}
$$


for any Lipschitz function $g$. Indeed, this follows from Theorem 4.4 applied to $f(x)=x g(x)$.

DEFINITION. A bounded non-negative measure $d L(x)$ is called a weak solution of $(6.12)$ if (6.13) holds for any continuously differentiable function $g(x)$ with uniformly bounded derivative.

THEOREM 6.4. Under the assumptions of Theorem 6.1 the measure $d L(x)$ is a weak solution of (6.12).

Proof. We first establish (6.13) in case

$$
\left|g^{\prime}(x)\right|+\left|g^{\prime \prime}(x)\right| \leq C, \quad g(x) \text { monotone increasing. }
$$

The starting point is the assertion that

$$
\frac{d}{d t} \int_{0}^{\infty} x g(x) n(x, t) \rightarrow 0 \text { if } t \rightarrow \infty
$$

To prove it we set

$$
\tilde{S}_{2}(t)=\int_{0}^{\infty} x g(x) n(x, t) d x
$$

and proceed as in the proof of Lemma 5.3. By Lemma 6.2

$$
\lim _{t \rightarrow \infty} \tilde{S}_{2}(t) \text { exists. }
$$

We next claim that

$$
\frac{d^{2} \tilde{S}_{2}(t)}{d t^{2}} \leq C
$$

To prove it we use Theorem 4.4 with $f(x)=x g(x)$ to get:

$$
\begin{aligned}
\frac{d \tilde{S}_{2}(t)}{d t}= & \frac{1}{2} \int_{0}^{\infty} d x \int_{0}^{\infty} d \xi K(x, \xi) x \xi n(x, t) n(\xi, t) \\
& \times\left[\frac{g(x+\xi)-g(x)}{\xi}+\frac{g(x+\xi)-g(\xi)}{x}\right] \\
+ & \int_{0}^{\infty} d x \int_{x}^{\infty} B(\xi, x) n(\xi, t) x(g(x)-g(\xi)) d \xi \equiv J_{1}(t)+J_{2}(t),
\end{aligned}
$$

and continue to differentiate once more in $t$ Consider first the case $B=0$. Then we can proceed similarly to the proof of $(5.13)$ with $f(x, c) / x$ in (5.16) replaced by

$$
f(x)=K(x, \xi)\left[\frac{g(x+\xi)-g(x)}{\xi}+\frac{g(x+\xi)-g(\xi)}{x}\right]
$$

where $\xi$ is viewed as a parameter. Using (5.2)(b) and the assumptions on $g$ in (6.14) we easily deduce that

$$
\frac{d^{2} \tilde{S}_{2}}{d t^{2}} \leq C
$$


If $B(x, \xi) \not \equiv 0$ then the additional terms in $d^{2} \check{S}_{2} / d t^{2}$ are also bounded, as can easily be shown.

Having proved $(6.16),(6.17)$, we can now quickly prove (6.15). Indeed, if (6.15) is not true then there is a sequence $t_{n} \rightarrow \infty$ such that

$$
\beta_{n} \equiv \frac{d \tilde{S}_{2}\left(t_{n}\right)}{d t} \rightarrow \beta \neq 0 \text { if } n \rightarrow \infty
$$

It follows that

$$
\begin{aligned}
\tilde{S}_{2}\left(t_{n}-\beta_{n} \epsilon\right)-\tilde{S}_{2}\left(t_{n}\right) & =-\beta_{n}^{2} \epsilon+\frac{1}{2} \beta_{n}^{2} \epsilon^{2} \tilde{S}_{2}^{\prime \prime}\left(\tilde{t}_{n}\right) \\
& \leq-\beta_{n}^{2} \epsilon+C \epsilon^{2}<-\frac{1}{2} \beta^{2} \epsilon
\end{aligned}
$$

if $\epsilon$ is positive and small and $n$ is large; this is a contradiction to (6.16).

So far we have proved that the left-hand side of (6.18) converges to zero as $t \rightarrow \infty$. Next we evaluate the right-hand side of $(6.18)$ as $t \rightarrow \infty$. By Theorem 6.1

$$
\begin{aligned}
J_{2}(t) & =\int_{0}^{\infty} \xi n(\xi, t) d \xi\left[\frac{1}{\xi} \int_{0}^{\xi} B(\xi, x) x(g(x)-g(\xi)) d x\right] \\
& \rightarrow \int_{0}^{\infty} d L(\xi) \int_{0}^{\xi} B(\xi, x) \frac{x}{\xi}(g(x)-g(\xi)) d x .
\end{aligned}
$$

To evaluate $J_{1}(t)$ introduce the functions

$$
\begin{aligned}
F(x, t) & =\int_{0}^{\infty} d \xi K(x, \xi) \xi n(\xi, t) \frac{g(x+\xi)-g(x)}{\xi} \\
F_{\infty}(x) & =\int_{0}^{\infty} d L(\xi) K(x, \xi) \frac{g(x+\xi)-g(x)}{\xi} .
\end{aligned}
$$

The family of functions $\{F(x, t), t>0\}$ is uniformly bounded and equicontinuous, and, by Theorem 6.1,

$$
F(x, t) \rightarrow F_{\infty}(x) \text { if } t \rightarrow \infty
$$

It follows that the convergence is uniform in any boundary interval $0 \leq x \leq R$. Using also Lemma 5.2, we easily deduce that

$$
\int_{0}^{\infty} x n(x, t)\left[F(x, t)-F_{\infty}(x)\right] d x \rightarrow 0 \text { if } n \rightarrow \infty .
$$

Since, by Theorem 6.1 ,

$$
\int_{0}^{\infty} d x n(x, t) F_{\infty}(x) \rightarrow \int_{0}^{\infty} d L(x) F_{\infty}(x)
$$


we conclude that

$$
\int_{0}^{\infty} x n(x, t) F(x, t) d x \rightarrow \int_{0}^{\infty} d L(x) F_{\infty}(x)
$$

if $t \rightarrow \infty$. Combining this result with (6.19), we deduce that the right-hand side of (6.18) converges to the left-hand side of (6.13), and since the left-hand side of (6.18) converges to zero, the equality $(6.13)$ follows.

So far we have assumed that $g$ satisfies (6.14). However by approximation we can establish (6.13) for any monotone increasing $g$ with first bounded and continuous derivative. Finally, if $g$ is not monotone increasing, we can decompose it into a difference of two monotone functions, and this completes the proof of Theorem 6.4.

Remark 6.1. If $n_{0}(x)$ is a stationary solution then $n(x, t)=n_{0}(x)$ and so

$$
\int_{0}^{\infty} n(x, c, t) d c=n_{0}(x)
$$

It follows that

$$
\int_{0}^{\infty} \int_{0}^{\infty} g(x) x n(x, c, t) d x d c=\int_{0}^{\infty} g(x) x n_{0}(x) d x
$$

and

$$
x n(x, c, t) d x d c \rightarrow \delta\left(c-c_{\infty}\right)\left(x n_{0}(x) d x\right)
$$

Remark 6.2. It was observed by Carr [2] that if $K$ and $B$ are related by

$$
K(x, y) Q(x) Q(y)=B(x+y, y) Q(x+y)
$$

where $Q$ is a positive function, then

$$
n_{\infty}(x)=e^{\lambda x} Q(x)
$$

is an equilibrium solution. Dubovskii and Stewart [5] proved that if $K(x, y)$ and $B(x, y)$ are linear functions then $n(x, t)$ converges to an equilibrium solution $n_{\infty}(x)$; their methods are entirely different from the methods of the present paper.

Acknowledgement. (1) We would like to thank David Ross from Eastman Kodak for suggesting the problems studied in this paper.

(2) The first author is partially supported by the National Science Foundation Grant DMS \#94-01251. The second author is partially supported by AFOSR through contract number F49620-95-1-0113 and by NSF through grant number DMS-9622555. 
Acknowledgement and Disclaimer. Effort sponsored by the Air Force Office of Scientific Research, Air Force Materials Command, USAF, under grant no. F49620-95-1. The US Government is authorized to reproduce and distribute reprints for governmental purposes notwithstanding any copyright notation thereby. The view and conclusions contained herein are those of the authors and should not be interpreted as necessarily representing the official policies or endorsements, either expressed or interpreted, of the Air Force Office of Scientific Research or the US Government.

\section{REFERENCES}

[1] O. Bruno, A. Friedman and F. Reitich, Asymptotic behavior for a coalescence problem, Trans. Amer. Math. Soc., 338 (1993), 133-158.

[2] J. Carr, Asymptotic behavior of solutions to the coagulation-fragmentation equations I. The strong fragmentation case, Proc. Royal Soc. Edinburgh, Sect. A 121 (1992), 231-244.

[3] P.B. Dubovskii, Mathematical Theory of Coagulation, Lecture Notes Series Number 23, Research Institute of Mathematics, Global Analysis Research Center, Seoul National University, Seoul, Korea (1994).

[4] P.B. Dubovskii and I.W. Stewart, Existence, uniqueness and mass conservation for the coagulationfragmentation equation, Math. Methods Appl. Sciences, 19 (1996), 571-591.

[5] P.B. Dubovskii and I.W. Stewart, Trend to equilibrium for the coagulation-fragmentation equation, Math. Methods Appl. Science, 19 (1996), 761-772.

[6] N. Dunford and J.T. Schwartz, Linear Operators, Part I, 4th edition, Interscience, New York (1967).

[7] A. Friedman, Generalized Functions, Prentice-Hall, Englewood Cliffs, N.J. (1963).

[8] A. Friedman, Mathematics in Industrial Problems, Part 10, in IMA Volume Series, Springer-Verlag, to appear.

[9] A. Friedman and F. Reitich. A hyperbolic inverse problem arising in the evolution of combustion aerosol. Archive Rat. Mech. Anal., 110 (1990), 313-350.

[10] Z.A. Melzak, A scalar transport equation, Trans. Amer. Math. Soc., 85 (1957), 547-560. 


\# Author/s Title

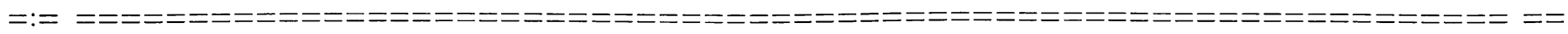

1398 B. Brighi \& M. Chipot, Approximation of infima in the calculus of variations

1399 G. Avalos, Concerning the well-posedness of a nonlinearly coupled semilinear wave and beam-like equation

1400 R. Lipton, Variational methods, bounds and size effects for composites with highly conducting interface

1401 B.T. Hayes \& P.G. LeFloch, Non-classical shock waves in scalar conservation laws

1402 K.T. Joseph \& P.G. LeFloch, Boundary layers in weak solutions to hyperbolic conservation laws

1403 Y. Diao, C. Ernst, \& E.J.J. Van Rensburg, Energies of knots

1404 Xiaofeng Ren, Multi-layer local minimum solutions of the bistable equation in an infinite tube

1405 Vlastimil Pták, Krylov sequences and orthogonal polynomials

1406 T. Aktosun, M. Klaus, \& C. van der Mee, Factorization of scattering matrices due to aprtitioning of potentials in one-dimensional Schrödinger-type equations

1407 C.-S. Man \& R. Paroni, On the separation of stress-induced and texture-induced birefringence in acoustoelasticity

1408 D.N. Arnold, R.S. Falk, \& R. Winther, Preconditioning discrete approximations of the Reissner-Mindlin plate model

1409 M.A. Kouritzin, On exact filters for continuous signals with discrete observations

1410 R. Lipton, The second Stekloff eigenvalue and energy dissipation inequalities for functionals with surface energy

1411 R. Lipton, The second Stekloff eigenvalue of an inclusion and new size effects for composites with imperfect interface

1412 W. Littman \& B. Liu, The regularity and singularity of solutions of certain elliptic problems on polygonal domains

1413 C.R. Collins, Spurious oscillations are not fatal in computing microstructures

1414 M.A. Horn, Sharp trace regularity for the solutions of the equations of dynamic elasticity

1415 A. Friedman, B. Hu \& Y. Liu, A boundary value problem for the Poisson equation with multi-scale oscillating boundary

1416 P. Bauman, D. Phillips \& Q. Tang, Stable nucleation for the Ginzburg-Landau system with an applied magnetic field

1417 J.M. Berg, A strain profile for robust control of microstructure using dynamic recrystallization

1418 P. Klouček, Toward the computational modeling of nonequilibrium thermodynamics of the Martensitic transformations

1419 S. Chawla \& S.M. Lenhart, Application of optimal control theory to in Situ bioremediation

1420 B. Li \& M. Luskin, Nonconforming finite element approximation of crystalline microstructure

1421 H. Kang \& J.K. Seo, Inverse conductivity problem with one measurement: Uniqueness of balls in $\mathbb{R}^{3}$

1422 Avner Friedman \& Robert Gulliver, Organizers, Mathematical modeling for instructors, July 29 - August 16, 1996

1423 G. Friesecke, Pair correlations and exchange phenomena in the free electron gas

1424 Y.A. Li \& P.J. Olver, Convergence of solitary-wave solutions in a perturbed Bi-Hamiltonian dynamical system

I. Compactons and Peakons II. Complex Analytic Behavior III. Convergence to Non-Analytic Solutions

1425 C. Huang, On boundary regularity of vortex patches for 3D incompressible euler systems

1426 C. Huang, A free boundary problem with nonlinear jump and kinetics on the free boundaries

1427 X. Chen, C. Huang \& J. Zhao, A nonlinear parabolic equation modeling surfactant diffusion

1428 A. Friedman \& B. Hu, Optimal control of chemical vapor deposition reactor

1429 A. Friedman \& B. Hu, A non-stationary multi-scale oscillating free boundary for the Laplace and heat equations

$1430 \mathrm{X}$. Chen, Existence, uniqueness, and asymptotic stability of traveling waves in nonlocal evolution equations

$1431 \mathrm{~J}$. Yong, Finding adapted solutions of forward-backward stochastic differential equations - Methods of continuation

1432 J. Yong, Linear forward-backward stochastic differential equations

1433 D.A. Dawson \& M.A. Kouritzin, Invariance principles for parabolic equations with random coefficients

1434 R. Lipton, Energy minimizing configurations for mixtures of two imperfectly bonded conductors

1435 D.C. Dobson \& F. Santosa, Nondestructive evaluation of plates using Eddy current methods

1436 W. Littman \& B. Liu, On the spectral properties and stabilization of acoustic flow

1437 S. Sarkar \& S. Sundar Sarkar, Normal distribution as a method for data replication in a parallel data server

1438 S. Sarkar \& S. Sundar Sarkar, Parallel view materialization with dynamic load balancing: A graph theoretic approach

1439 S. Sarkar \& S. Sundar Sarkar, Internet and relational databases in a multi-tier client/server model

1440 J. Liang \& S. Subramaniam, Numerical computing of "molecular electrostatics through boundary integral equations

$1441 \mathrm{~J}$. Wu, Inviscid limits and regularity estimates for the solutions of the 2-D dissipative quasi-geostrophic equations

1442 P. Constantin \& J. Wu, Statistical solutions of the Navier-Stokes equations on the phase space of vorticity and the inviscid limits

1443 M.A. Kouritzin, Stochastic processes and perturbation problems defined by parabolic equations with a small parameter

1444 M.A. Kouritzin, Approximations for singularly perturbed parabolic equations of arbitrary order

1445 A. Novick-Cohen Triple junction motion for Allen-Cahn/Cahn-Hilliard systems

1446 P. Klouček, Approximations of the laminated microstructures 
1447 S. Sarkar \& S.S. Sarkar, A graph theoretic approach for parallel view materialization with dynamic load balancing

1448 S. Chawla, A minmax problem for parabolic systems with competitive interactions

1449 B. Luong \& F. Santosa, Quantitative imaging of corrosion in plates by Eddy current methods

1450 R. Jordan \& B. Turkington, Ideal magnetofluid turbulence in two dimensions

1451 M. Fels \& P.J. Olver, Moving coframes. I. A practical algorithm

1452 S.Y. Maliassov, On the Schwarz alternating method for eigenvalue problems

1453 R. Lipton, Design of particle reinforced heat conducting composites with interfacial thermal barriers

1454 J. Berg, A. Yezzi, \& A. Tannenbaum, Phase transitions, curve evolution, and the control of semiconductor manufacturing processes

1455 G. Avalos \& I. Lasiecka, Uniform decay rates of solutions to a structural acoustics model with nonlinear dissipation

1456 M. Nitsche, Siemens/IMA technical report

1457 L. Wang, J.A. Cox, \& A. Friedman, Model analysis of homogeneous optical waveguides by boundary integral method

1458 C.P. Fung \& S. Lototsky, Nonlinear filtering: Separation of parameters and observations using Galerkin approximation and Wiener chaos decomposition

1459 S. Northshield, Several proofs of Ihara's theorem

1460 T. Aktosun, M. Klaus \& C. van der Mee, Wave scattering in one dimension with absorption

1461 F. Santosa, M. Vogelius, \& J.-M. Xu, An effective nonlinear boundary condition for a corroding surface. Identification of the damage based on electrostatic data

1462 J. Wu, Well-posedness of a semilinear heat equation with weak initial data

1463 J. Wu, Quasi-geostrophic type equations with weak initial data

1464 J. Ma \& J. Yong, Approximate solvability of forward-backward stochastic differential equations

1465 T.-P. Tsai, On Leray's self-similar solutions of the Navier-Stokes equations satisfying local energy estimates

1466 M.K. Gobbert, T.P. Merchant, L.J. Borucki, \& T.S. Cale, A multiscale simulator for low pressure chemical vapor deposition

1467 M.C. Tesi, E.J. Janse van Rensburg, E. Orlandini, \& S.G. Whittington, Torsion of polygons in $\mathcal{Z}^{3}$

1468 M. Grinfeld \& A. Novick-Cohen, The viscous Cahn-Hilliard equation: Morse decomposition and structure of the global attractor

1469 M. Mascagni, Polynomial versus matrix methods for leap-ahead in shift-register type pseudorandom number generators

1470 M. Mascagni, Parallel linear congruential generators with prime moduli

1471 B. Li \& M. Luskin, Approximation of a Martensitic laminate with varying volume fractions

1472 D. Yang, Stabilized schemes for mixed finite element methods with applications to elasticity and compressible flow problems

1473 P.J. Olver \& V.V. Sokolov, Integrable evolution equations on associative algebras

1474 A. Bondarenko, Singular structure of the fundamental solution of the transport equation

1475 S. Sarkar \& S.S. Sarkar, Views and data mining in a parallel data server

1476 P.E. Bigeleisen \& M. Cheney, Models for an anesthesia breathing circuit

1477 R. Lipton, Influence of interfacial surface conduction on the DC electrical conductivity of particle reinforced composites

1478 B. Cockburn \& C.-W. Shu, The local discontinuous Galerkin method for time-dependent convection-diffusion systems

1479 A. Friedman \& F. Reitich, Asymptotic Behavior of Solutions of Coagulation-Fragmentation Models

1480 A. Friedman \& C. Huang, Averaged Motion Approach to Brownian Dynamics

1481 T. Aktosun, M. Klaus \& C. van der Mee, Inverse Scattering in One-Dimensional Nonconservative Media

1482 X. Feng, Absorbing Boundary Conditions for Electromagnetic Wave Propagation

1483 J.E. Flaherty, R.M. Loy, M.S. Shephard, B.K. Szymanski, J.D. Teresco \& L.H. Ziantz, Adaptive Local Refinement with Octree Load-Balancing for the Parallel Solution of Three-Dimensional Conservation Laws

1484 D. Hömberg \& J. Sokolowski, Optimal Control of Laser Hardening

1485 M. Kružík, Numerical Approach to Double Well Problems

1486 S. Lototsky, Parameter Estimation for Stochastic Parabolic Equations: Asymptotic Properties of a Two-Dimensional Projection Based Estimate

1487 D.E. Norman, A Model for Chemically Reacting Fluid Flows: Weak Solutions and Global Attractors

1488 S. Sarkar \& S.S. Sarkar, Data Mining on Views in Relational Databases Using Bayesian Networks

1489 D. Yang, Dynamic Finite Element Methods for Second Order Parabolic Equations

1490 S. Evje \& K.H. Karlsen A Note on Viscous Splitting of Degenerate Convection-Diffusion Equations

1491 X. Feng, Analysis of A Domain Decomposition Method for The Nearly Elastic Wave Equations Based on Mixed Finite Element Methods

1492 B. Cockburn \& C.-W. Shu, The Runge-Kutta Discontinuous Galerkin Method for Conservation Laws V: Multidimensional Systems

1493 P. Korman \& Y. Li, On The Exactness of an S-Shaped Bifurcation Curve

1494 M. Kružík, DiPerna-Majda Measures and Uniform Integrability 